distracting them from other learning objectives. There is specifically a lack of research regarding medical students' own beliefs and attitudes towards sim man dying. This pilot study explores whether final year medical students feel it is acceptable for sim man to die and to gain insight into their attitudes and beliefs regarding death in simulation.

Methods A paper questionnaire was distributed to final year medical students on placement at Swindon Academy in December 2016. The survey included quantitative dichotomous questions with white space for students' comments. The free text comments regarding concerns and benefits from sim man dying were analysed using thematic analysis and coding.

Results A total of 34 out of 35 students completed the questionnaire. $97 \%$ of final year students felt they should have end of life simulation scenarios. $88 \%$ of final year students felt that sim man should be allowed to die in acute scenarios.

94\% said there were benefits of sim man dying during simulations. The four themes within this included preparing to deal with death; experiencing death in a safe environment; realism of simulation and practicalities/skills regarding death.

$27 \%$ of final year students had concerns about sim man dying. The three main themes were emotional distress; feeling underprepared and negatively impacting on confidence.

Conclusions The large majority of final year medical students feel that sim man should be allowed to die in both end of life and acute simulations. Educators should not assume sim man dying is too distressing for medical students and should consider utilising this as an undergraduate learning tool for dealing with death but providing a sensitive and supportive environment with a focus on debrief is essential.

\section{P-135 FROM DIAGNOSIS TO DEATH - A PILOT OF INTER- PROFESSIONAL PALLIATIVE CARE SIMULATION FOR MEDICAL AND NURSING STUDENTS}

\footnotetext{
${ }^{1,2}$ Joseph Hartland, ${ }^{1,2}$ Francesca Bold, ${ }^{3}$ Lorraine Whatley. ${ }^{1}$ Swindon Undergraduate Academy, Great Western Hospital, Swindon, UK; ${ }^{2}$ Bristol University, Bristol, UK; ${ }^{3}$ Oxford Brookes Nursing School, UK
}

\subsection{6/bmjspcare-2017-00133.134}

Background Effective palliative care is delivered in the multidisciplinary team (MDT) setting. ${ }^{1}$ Despite this the majority of under graduate/preregistration teaching takes place in a unidisciplinary environment.

In the 2015-2016 Academic year Swindon Academy and Oxford Brooke's Nursing school sought to address this issue through a pilot program of MDT high-fidelity simulations focused on a patient in the last six months of life.

This project aims to improve undergraduates' confidence in the diagnosis and management of palliative problems, as well as their understanding of each other's roles and ability to work confidently in a team.

Method 44 final year Medical students and second year Nursing students undertook four scenarios that followed a patient (an actor and finally a Sim Man) from diagnosis of a terminal cancer, breaking bad news, diagnosing the dying patient and management of symptoms at the end of life.

Confidence was self-assessed pre and post session on an anonymised 10 point Likert scale. Data collected was analysed for statistical significance using the Mann-Whitney $U$ test.

Results The session was rated 8.7 overall and every category of self-confidence assessment showed an overall mean improvement. All areas of self-assessment showed statistically significant improvements in pre and post scores, including in 'awareness of team member roles' and 'communicating with patients and families'.

Conclusion Palliative care in a simulated environment appears to be an effective teaching tool. Inter-professional teaching seems to improve awareness of professionals' roles, thereby improving the care provided by teams of new junior doctors and nurses.

An observational study is now underway on whole-day simulation workshops which we hope will further support our argument that inter-professional simulation teaching can effectively deliver palliative care education and improved ability to work in a MDT.

\section{REFERENCE}

1. Royal College of Physicians, National Care of the Dying Audit for hospitals, Eng land, 2014. [Online] Available at:https://www.rcplondon.ac.uk/file/903/download? token=oregrc-n Accessed:27/6/16.

\section{P-136 PROVIDING PALLIATIVE CARE TO THE WORLD'S POOREST: A SERVICE REVIEW OF A COMMUNITY PALLIATIVE CARE PROGRAMME IN MULANJE MISSION HOSPITAL, MALAWI}

${ }^{1}$ Rebecca Butler, ${ }^{2} J o s e p h$ Hartland. ${ }^{1}$ Bristol University, UK; ${ }^{2}$ Swindon Academy Undergraduate Department, Swindon, UK

\subsection{6/bmjspcare-2017-00133.135}

Background Despite a huge need for palliative care in SubSaharan Africa it is estimated that only $5 \%$ of those requiring palliative care in Sub-Saharan Africa receive it. ${ }^{1}$ One hospital providing an outpatient palliative care service since is Mulanje Mission Hospital (MMH) in southern Malawi. The clinic currently services 462 patients between 3 nurses, with appointments offered 2-3 times per year. Due to geographical challenges one home visit can take up to 5 hours to complete.

As part of Swindon Academy's External Student Selected Component program a field trip to $\mathrm{MMH}$ was undertaken in 2016. Approval from the senior board of $\mathrm{MMH}$ this service evaluation aimed to analyse the population demographic and look for any patterns of mortality.

Methods 100 outpatient palliative care records were examined from a 2 year sample to determine the last point of contact with the patient and the number of years they spent in the programme, with Chi-squared tests used to assess significance. Results Of the 100 patients 45 died within one year of first contact. $32 \%$ of these deaths were due to Kaposi Sarcoma (KS), despite KS making up 57\% of the primary diagnosis. Conversely $53 \%$ of deaths within one year were due to other non-KS cancers, most commonly cervical. It was statistically significant that a patient was more likely to be alive with KS than with other type of cancer $(p=0.0000162)$.

Conclusions To correctly prioritise the needs of palliative care patients in low resource settings there needs to be identification of patients who are most likely to require intensive input. Anticipating the need for end of life care may allow increased clinic reviews and ultimately better symptom management to be prioritised.

\section{REFERENCE}

1. Harding, R Powell, RA Downing, J Connor, et al. Generating an African palliative care evidence base: the context, need, challenges, and strategies. I Pain Symptom Manage 2008;36(3):304-9. 